\title{
Tests and relevancies for the hypotheses of an orthogonal family in a model with orthogonal block structure
}

\author{
Dário Ferreira $^{\mathrm{a}}$, Sandra S. Ferreira ${ }^{\mathrm{a}}$, Célia Nunes ${ }^{\mathrm{a}}$ and João T. Mexia ${ }^{\mathrm{b}}$ \\ ${ }^{a}$ Department of Mathematics and Center of Mathematics and Applications, University of \\ Beira Interior, Portugal; ${ }^{b}$ Center of Mathematics and its Applications, Faculty of Science \\ and Technology, New University of Lisbon, Portugal
}

\section{ARTICLE HISTORY}

Compiled October 27, 2019

\begin{abstract}
A model has an orthogonal block structure if it has, as covariance matrix, a linear combination of pairwise orthogonal projection matrices, that add up to the identity matrix. The range space of these matrices are associated to hypotheses of an orthogonal family.

In this paper we show how to obtain tests for these hypotheses when normality is assumed and how to consider their relevance when normality is discarded. Besides the notion of relevance, we formulate hypotheses in a general way that may be applied to models with orthogonal block structure, whose factors may have fixed and/or random effects. The results are applied to prime basis factorial models and an example is presented.
\end{abstract}

\section{KEYWORDS}

Inference; mixed models; variance components; hypotheses relevancies; non-normality; prime basis factorials

\section{Introduction}

Consider the linear model

$$
\boldsymbol{Y}=\sum_{i=0}^{w} \boldsymbol{X}_{i} \boldsymbol{\beta}_{i},
$$

where $\boldsymbol{Y}$ is a vector of $N$ random variables $Y_{1}, \ldots, Y_{N}$. Model (1) will be a mixed model if $\boldsymbol{\beta}_{0}$ is a fixed vector and the $\boldsymbol{\beta}_{1}, \ldots, \boldsymbol{\beta}_{w}$ are random and independent, with null mean vectors, covariance matrices $\boldsymbol{V}\left(\boldsymbol{\beta}_{i}\right)=\sigma_{i}^{2} \boldsymbol{I}_{c_{i}}, i=1, \ldots, w$, and null cross covariance matrices, $\boldsymbol{V}\left(\boldsymbol{\beta}_{i} ; \boldsymbol{\beta}_{\ell}\right)=\mathbf{0}_{c_{i} \times c_{l}}$ for all $i \neq \ell$. The model has mean vector $\boldsymbol{\mu}=\boldsymbol{X}_{0} \boldsymbol{\beta}_{0}$ and covariance matricx

$$
\boldsymbol{V}(\boldsymbol{Y})=\sum_{i=1}^{w} \sigma_{i}^{2} \boldsymbol{M}_{i}
$$


with $\boldsymbol{M}_{i}=\boldsymbol{X}_{i} \boldsymbol{X}_{i}^{\top}, i=1, \ldots, w$. So $\boldsymbol{M}_{i}$ is the $N \times N$ relation matrix for the $i$-th random factor. That is, its $(a, b)$-entry is equal to 1 if this factor has the same level on units $a$ and $b$, otherwise it is equal to 0 , see $[1,2]$.

In models in which the relation matrices $\boldsymbol{M}_{1}, \ldots, \boldsymbol{M}_{w}$ commute, we have, see [3],

$$
\boldsymbol{M}_{i}=\sum_{j=1}^{m} b_{i, j} \boldsymbol{Q}_{j},
$$

$i=1, \ldots, w$, where $\boldsymbol{Q}_{1}, \ldots, \boldsymbol{Q}_{m}$ are known orthogonal symmetric idempotent matrices of order $N$, summing to the identity matrix $\boldsymbol{I}_{N}$, see [4]. Matrices $\boldsymbol{Q}_{j}, j=1, \ldots, m$, are also known as pairwise orthogonal projection matrices. Then the covariance matrices may be rewritten as

$$
\boldsymbol{V}(\boldsymbol{\gamma})=\sum_{j=1}^{m} \gamma_{j} \boldsymbol{Q}_{j}
$$

with $\boldsymbol{\gamma}=\left(\gamma_{1}, \ldots, \gamma_{m}\right)^{\top}$ and $\gamma_{j}=\sum_{i=1}^{w} b_{i, j} \boldsymbol{Q}_{j}, j=1, \ldots, m$. If matrix $\boldsymbol{B}=\left[b_{i, j}\right]$ is invertible, $\boldsymbol{V}(\gamma)$ will be the positive semi-definite linear combinations of $\boldsymbol{Q}_{1}, \ldots, \boldsymbol{Q}_{m}$ and the models will have orthogonal block structure, OBS, see $[5,6]$.

With $\nabla_{j}=R\left(\boldsymbol{Q}_{j}\right)$ the range space of $\boldsymbol{Q}_{j}, j=1, \ldots, m$, such a model will be associated to the orthogonal partition

$$
\mathbb{R}^{N}=\boxplus_{j=1}^{m} \nabla_{j}
$$

see [4], and $\boldsymbol{Y}_{j}=\boldsymbol{Q}_{j} \boldsymbol{Y}, j=1, \ldots, m$, will have covariance matrix $\gamma_{j} \boldsymbol{Q}_{j}$ and mean vector

$$
\boldsymbol{\mu}_{j}=\boldsymbol{Q}_{j} \boldsymbol{\mu},
$$

with $\boldsymbol{\mu}$ the mean vector of $\boldsymbol{\mu}_{1}, \ldots, \boldsymbol{\mu}_{m}$ and non-centrality parameter

$$
\delta_{j}=\frac{1}{\gamma_{j}}\left\|\boldsymbol{\mu}_{j}\right\|^{2}
$$

$j=1, \ldots, m$.

In the next section we show how to obtain tests for the hypotheses of the orthogonal family associated to $\nabla_{j}$, and how to consider their relevance when normality is discarded. In Section 3 we apply the obtained results to prime basis factorials. The used methodology will allow the study of a larger number of interactions simultaneously compared with the classical approach, see [7]. In Section 4 we present a concrete example. Finally, in Section 5, we present some final remarks.

\section{Tests hypotheses and relevancies}

Model (1) may be written as

$$
Y=Z+e,
$$


where $\boldsymbol{Z}=\sum_{i=0}^{w-1} \boldsymbol{X}_{i} \boldsymbol{\beta}_{i}$ and $\boldsymbol{e}$ is the error vector, with null mean vector, covariance matrix $\sigma^{2} \boldsymbol{I}_{n}$ and is independent of $\boldsymbol{Z}$, which has mean vector $\boldsymbol{\mu}$ and covariance matrix $\sum_{j=1}^{m} \delta_{j} \boldsymbol{Q}_{j}$, where $\delta_{j}=\gamma_{j}-\sigma^{2} \geq 0, j=1, \ldots, m$.

We can now assume the subspaces $\nabla_{j}, j=1, \ldots, m$, in (4), associated to the hypotheses

$$
H_{0, j}: \delta_{j}=\gamma_{j}-\sigma^{2}=0
$$

$j=1, \ldots, m$. When $H_{0, j}$ holds, $\boldsymbol{Q}_{j} \boldsymbol{Z}$ will have null mean vector, as well as null covariance matrix, so that

$$
\operatorname{pr}\left(\boldsymbol{Q}_{j} \boldsymbol{Y}=\boldsymbol{Q}_{j} \boldsymbol{e}\right)=\operatorname{pr}\left(\boldsymbol{Q}_{j} \boldsymbol{Z}=\mathbf{0}\right)=1 .
$$

If we assume that the $H_{0, j}$, with $j \in \mathcal{C}$, hold and that $\boldsymbol{e}$ is normal, we will have, for the $H_{0, j^{\prime}}$ with $j^{\prime} \notin \mathcal{C}$, the test statistics

$$
F_{j^{\prime}}=\frac{g}{g_{j^{\prime}}} \frac{S_{j^{\prime}}}{S} ; j^{\prime} \notin \mathcal{C}
$$

where $S_{j^{\prime}}=\left\|\boldsymbol{Q}_{j^{\prime}} \boldsymbol{Y}\right\|^{2}, g_{j^{\prime}}=\operatorname{rank}\left(\boldsymbol{Q}_{j^{\prime}}\right), g=\sum_{j \in \mathcal{C}} g_{j}$ and $S$ is the sum of squares of $\boldsymbol{e}$. These tests have a central $\mathrm{F}$ distribution with $g_{j^{\prime}}$ and $g$ degrees of freedom. Moreover, $S$ is the product by $\sigma^{2}$ of a central chi-square, with $g$ degrees of freedom. Furthermore, with $U=\frac{S}{g}$ and $s_{j^{\prime}}=\frac{S_{j^{\prime}}}{g_{j^{\prime}}}, j \notin \mathcal{C}$, we have

$$
F_{j^{\prime}}=\frac{s_{j^{\prime}}}{U}, \quad j^{\prime} \notin \mathcal{C}
$$

On the other hand, when $H_{0, j^{\prime}}, j^{\prime} \notin \mathcal{C}$, does not hold, we may have

$$
\left\{\begin{array}{l}
\sigma^{2}<\gamma_{j^{\prime}} \\
0<\delta_{j^{\prime}}
\end{array}\right.
$$

While $\sigma^{2}<\gamma_{j^{\prime}}$ refers to random effects models, $0<\delta_{j^{\prime}}$ refers to fixed effects models. Nevertheless both may be considered in mixed models. So, this hypotheses formulation holds for three classes of models: fixed, random and mixed. Moreover, considering the hypotheses $H_{0, j^{\prime}}, j^{\prime} \notin \mathcal{C}$ against $H_{1, j^{\prime}}, F_{j^{\prime}}=\frac{s_{j^{\prime}}}{U}, j^{\prime} \notin \mathcal{C}$, points towards using $S_{j^{\prime}}$ to measure the relevance, $R_{j^{\prime}}$ of the alternative hypotheses. This leads to introducing the ANOVA profile $S_{j^{\prime}}, j^{\prime} \notin \mathcal{C}$.

We point out that if, for instance, for all $n$ considered treatments (combination of factor levels), we had $r$ replications, then $S$ would be the sum of the sums of squares of the residuals and we would have $g=n(r-1)$.

Up to now normality was assumed. However, if $Y$ has OBS, it is not needed to have normality because, in that case, we may reason as before and measure the relevance of the $H_{1, j^{\prime}}$ by the $S_{j^{\prime}}, j^{\prime} \notin \mathcal{C}$, even when $\mathcal{C}=\mathbf{0}$.

As pointed above, the $H_{0, j^{\prime}}, j^{\prime} \notin \mathcal{C}$, are not restricted to a class of models. So, in the next section, we will consider prime basis factorials. 


\section{Prime Basis Factorials}

In what follows we will use the $\bmod (r)$ arithmetic, in which the results in the usual arithmetic are replaced by the residuals of their division by $r$. We denote the sum, $a+b$, and the product, $a b$, in $\bmod (r)$ arithmetic, by $a+_{(r)} b$ and $(a b)_{(r)}$, respectively.

Consider now a model with $u$ factors and $p$ prime levels. Thus, there will be $n=p^{u}$ treatments.

With $x_{i}=0, \ldots, p-1, i=1, \ldots, u$, the level of the $i$-th factor, the treatments may be represented by $\boldsymbol{x}=\left(x_{1}, \ldots, x_{u}\right)$. So, we have the linear function

$$
L(\boldsymbol{x} \mid \boldsymbol{a})=a_{1} x_{1}+_{(p)} \cdots+_{(p)} a_{u} x_{u} .
$$

Note that there are $p^{u}$ of these functions of which

$$
q_{u}=\frac{p^{u}-1}{p-1}
$$

are reduced, having 1 as their first non null coefficients, see [8].

Let $\boldsymbol{a}_{i}, i=1, \ldots, q_{u}$, be the vector of coefficients of the $i-t h$ linear function $L_{i}(\boldsymbol{x})$. Taking

$$
j(\boldsymbol{x})=1+\sum_{h=1}^{u} x_{h} p^{h-1},
$$

as the index of treatments $\boldsymbol{x}$, we can associate to $L_{i}(\boldsymbol{x})$ the matrices

$$
B(i)=\left[b_{h, j}(i)\right],
$$

$i=1, \ldots, q_{u}$, with elements

$$
\left\{\begin{array}{ll}
b_{h, j}(i)=1, & \text { if } \quad L_{i}\left(\boldsymbol{x}_{j}\right)=h-1 \\
b_{h, j}(i)=0, & \text { if } \quad L_{i}\left(\boldsymbol{x}_{j}\right) \neq h-1
\end{array} .\right.
$$

Let

$$
\left(L_{i} ; h\right)=\left\{\boldsymbol{x} ; L_{i}(\boldsymbol{x})=h-1\right\},
$$

$h=1, \ldots, p, i=1, \ldots, q_{u}$. The totals for these sets of treatments will be the components of $\boldsymbol{B}(i) \boldsymbol{Y}, i=1, \ldots, q_{u}$. It may be shown, see [9], that the sum of squares associated to $L_{i}, i=1, \ldots, q_{u}$ is given by

$$
S_{i}=p^{u-1}\|\boldsymbol{A}(i) \boldsymbol{Y}\|^{2},
$$

where $\boldsymbol{A}(i)=\boldsymbol{C}_{p} \boldsymbol{B}(i), i=1, \ldots, q_{u}$, with $\boldsymbol{C}_{p}$ obtained by deleting the first row, which is equal to $\frac{1}{\sqrt{p}} \mathbf{1}_{p}^{\top}$, of a $j \times p$ orthogonal matrix, where $\mathbf{1}_{p}$ is a vector of ones with length $p$. Moreover, it may be shown that the row vectors of $\frac{1}{p^{\frac{u-1}{2}}} \boldsymbol{A}(i)$ constitute an orthonormal basis to the subspace $\nabla(i), i=1, \ldots, q_{u}$, associated to $L(i)$.

The order of $L(i)$ will be the number of its non null coefficients minus 1 . If the order is $0, L(i)$ will be associated to the effects of the sole factor with one null coefficient, which 
will be 1 . If the order is 1 , it will be associated to a factorial interaction between the pair of factors with $m$ null coefficients, and so on. Usually the higher order interactions are used to estimate the errors. So, the $L(i)$ corresponding to these interactions will have indexes in $\mathcal{C}$ and we will have

$$
S=\sum_{i \in \mathcal{C}} S(i)
$$

as well as

$$
g=(p-1) \sharp \mathcal{C} .
$$

We will now consider recurrence relations. Let $P(u)$ be the set of vector coefficients, $\boldsymbol{a}$, for reduced linear functions, when there are $u$ factors. Then

$$
P(u+1)=\left\{\boldsymbol{\delta}_{u+1}\right\} \cup \bigcup_{h=1}^{p-1} P(u)+h \boldsymbol{\delta}_{u+1},
$$

where $\boldsymbol{\delta}_{u+1}=(0, \ldots, 1)$ has as non null component the $(u+1)$-th component, which will be 1 , and

$$
P(u)+h \boldsymbol{\delta}_{u+1}=\left\{\boldsymbol{v}+h \boldsymbol{\delta}_{u+1}, \quad \boldsymbol{v} \in P(u)\right\} .
$$

To extend the ordering of the reduced linear functions with $u$ factors to that of when we have $u+1$ factors we put

$$
\left\{\begin{array}{l}
i_{u+1}(\boldsymbol{a})=i_{u}(\boldsymbol{a}) ; \quad \boldsymbol{a} \in \mathcal{D}(u) \\
i_{u+1}\left(\boldsymbol{\delta}_{u+1}\right)=q_{u}+1 \\
i_{u+1}\left(\boldsymbol{a}+h \boldsymbol{\delta}_{u+1}\right)=i_{u}(\boldsymbol{a})+1+h q_{u} ; \quad h=1, \ldots, j-1, \quad \boldsymbol{a} \in \mathcal{D}(u)
\end{array},\right.
$$

with $\mathcal{D}(u)$ the set of vector coefficients when there are $u$ factors.

Furthermore, it may be seen that, with $i=i_{u}(\boldsymbol{a})$ and $i_{h}=i_{u+1}\left(\boldsymbol{a}+h \boldsymbol{\delta}_{u+1}\right)$, we have

$$
L_{i_{h}}\left(\boldsymbol{x}+h^{\prime} \boldsymbol{\delta}_{u+1}\right)=L_{i}(\boldsymbol{x})+{ }_{(p)}\left(h h^{\prime}\right)_{(p)} .
$$

We thus obtain the recurrence relation for matrices $\boldsymbol{B}_{i_{h}}(u+1)$ given by

$$
\boldsymbol{B}_{i_{h}}(u+1)=\left[\begin{array}{llll}
\boldsymbol{B}_{i}(u) & \boldsymbol{B}_{i}(u)+_{(p)} h \mathbf{1}_{p} \mathbf{1}_{p^{u}}^{\top} & \ldots & \boldsymbol{B}_{i}(u)+_{(p)}\left((p-1)_{p} h\right) \mathbf{1}_{p} \mathbf{1}_{p^{u}}^{\top}
\end{array}\right] .
$$

When $h=0$, we have $i_{h}=i$ and

$$
\boldsymbol{B}_{i}(u+1)=\mathbf{1}_{p}^{\top} \otimes \boldsymbol{B}_{i}(u) .
$$

Moreover, we also have

$$
\boldsymbol{B}_{q+1}(u+1)=\mathbf{0}_{p^{u}} \boldsymbol{I}_{p} \otimes \mathbf{1}_{p^{u}}^{\top} .
$$

We point out that $\boldsymbol{B}_{i}(u)$ is $p \times p^{u}$ and that $\boldsymbol{B}_{i}(u+1)$ is $p \times p^{u+1}$. 


\section{Example}

We will now consider a $3^{3}$ model, in which three factors, $A, B$ and $C$, with three levels each, cross. The data is presented in Table 1 .

Table 1. Data

\begin{tabular}{|c|ccc|ccc|ccc|}
\hline & \multicolumn{3}{|c|}{$A_{1}$} & \multicolumn{3}{c|}{$A_{2}$} & \multicolumn{3}{c|}{$A_{3}$} \\
\hline & $B_{1}$ & $B_{2}$ & $B_{3}$ & $B_{1}$ & $B_{2}$ & $B_{3}$ & $B_{1}$ & $B_{2}$ & $B_{3}$ \\
\hline$C_{1}$ & 0,33 & 0,67 & 0,33 & 2,67 & 2,67 & 1,33 & 2,00 & 1,00 & 2,00 \\
$C_{2}$ & 0,50 & 0,50 & 1,00 & 4,00 & 4,00 & 4,00 & 3,00 & 3,00 & 1,50 \\
$C_{3}$ & 0,67 & 1,33 & 1,33 & 2,67 & 5,33 & 5,33 & 4,00 & 2,00 & 2,00 \\
\hline
\end{tabular}

The observations vector is the corresponding vec. Matrices $\boldsymbol{B}$ are easy to obtain, using the results in the previous section. We indicate the reduced factors, corresponding to each matrix, writing it as $\boldsymbol{B}_{L_{i}}(h)$, instead of $\boldsymbol{B}_{i}$. So, with $\boldsymbol{J}_{3 \times 3}=\mathbf{1}_{3} \mathbf{1}_{3}^{\top}$ and using $\bmod (3)$ arithmetic, we get

$$
\begin{aligned}
& \boldsymbol{B}_{x_{1}}(1)=\boldsymbol{I}_{3},
\end{aligned}
$$

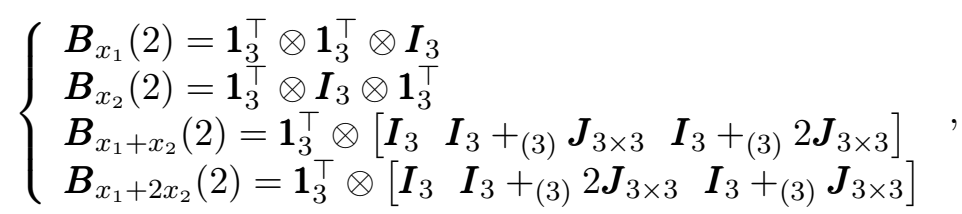

and

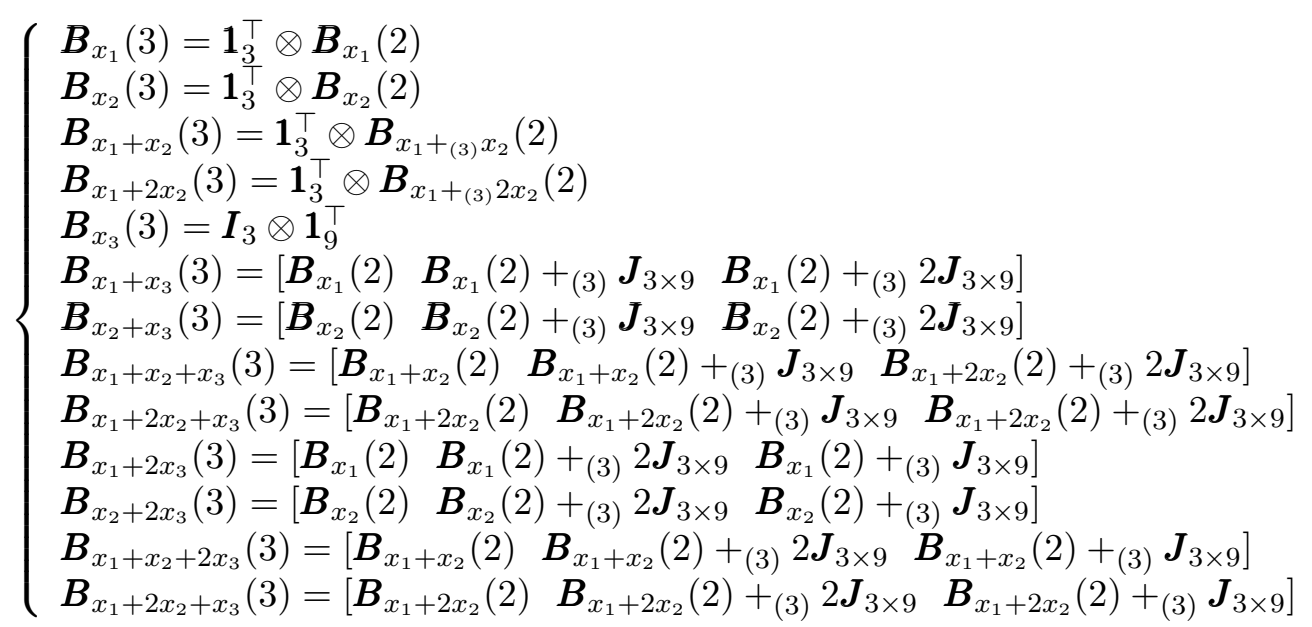

Now, considering the orthogonal matrix

$$
C^{*}=\left[\begin{array}{ccc}
\frac{1}{\sqrt{3}} & \frac{1}{\sqrt{3}} & \frac{1}{\sqrt{3}} \\
-\frac{1}{\sqrt{2}} & 0 & \frac{1}{\sqrt{2}} \\
-\frac{1}{\sqrt{6}} & -\frac{2}{\sqrt{6}} & \frac{1}{\sqrt{6}}
\end{array}\right],
$$


we obtain

$$
C_{3}=\left[\begin{array}{ccc}
-\frac{1}{\sqrt{2}} & 0 & \frac{1}{\sqrt{2}} \\
-\frac{1}{\sqrt{6}} & -\frac{2}{\sqrt{6}} & \frac{1}{\sqrt{6}}
\end{array}\right],
$$

removing the first row to $C^{*}$.

The sum of squares associated to $L_{i}, i=1, \ldots, 13$, indicated in (19), are, approximately, $S_{1}=654, S_{2}=13, S_{3}=67, S_{4}=8, S_{5}=2898, S_{6}=32, S_{7}=550, S_{8}=155$, $S_{9}=339, S_{10}=378, S_{11}=252, S_{12}=385, S_{13}=439$. Now, the relevance of the several alternative hypotheses may be measured by

$$
R_{i}=\frac{S_{i}}{\sum_{i=1}^{13} S_{i}} .
$$

It may be seen that there are great differences in the relevance of several hypotheses, with, for example, $R_{2}=0.0021$ and $R_{5}=0.4697$.

\section{Final remarks}

Testing null hypotheses significance may not be sufficient, because some of these may be of little relevance. In this paper we showed how to measure the relevance of the several null significant hypotheses, in models with orthogonal block structure. To do that we estimated the part of the total variation that corresponds to the projection of the mean vector on the range space of known orthogonal symmetric idempotent matrices of order $N$, summing to the identity matrix. We point out that normality is not needed and that the method is not restricted to a class of models. We presented a concrete example considering prime basis factorials.

\section{Acknowledgements}

This work was partially supported by the Portuguese Foundation for Science and Technology through the projects UID/MAT/00212/2019 and UID/MAT/00297/2019.

\section{References}

[1] Bailey RA. Orthogonal partitions in designed experiments. Designs, Codes and Cryptography 1996;8;45-77.

[2] Bailey RA, Association Schemes. Cambridge Studies in Advanced Mathematics 2004;84 Cambridge: Cambridge University Press.

[3] Bailey RA, Ferreira SS, Ferreira D, Nunes C. Estimability of Variance Components when all Model Matrices Commute. Linear Algebra and its Applications 2015;492;144-160.

[4] Seely J. Quadratic subspaces and completeness. Ann. Statist. 1971;42;2;710-721.

[5] Nelder JA. The analysis of randomized experiments with orthogonal block structure. I. Block structure and the null analysis of variance. Proceedings of the Royal Society (London), Series A 1965a;273;147-162.

[6] Nelder JA. The analysis of randomized experiments with orthogonal block structure. II. Treatment structure and the general analysis of variance. Proceedings of the Royal Society (London), Series A, 1965b;273;163-178. 
[7] Carvalho F. Strictly associated models, prime basis factorials: an application. Discussiones Mathematicae Probability and Statistics 2011;31;77-86

[8] Oliveira S. Delineamento regressional mltiplo para um factorial de base prima estritamente associado a uma lgebra de Jordan comutativa [dissertation]. Universidade Nova de Lisboa; 2018. Available from: http://hdl.handle.net/10362/45217

[9] Mexia JT. Standardized Orthogonal Matrices and the Decomposition of the sum of Squares for Treatments. Trabalhos de Investigação 2, Departamento de Matemática FCTUNL 1988. 